\title{
Application of Artificial Intelligence in Early Gastric Cancer Diagnosis
}

\author{
Zili Xiao Danian Ji Feng Li Zhengliang Li Zhijun Bao \\ Department of Gastroenterology, Huadong Hospital Affiliated to Fudan University, Shanghai, China
}

\section{Keywords}

Artificial intelligence $\cdot$ Convolutional neural network · Early gastric cancer · Esophagogastroduodenoscopy · Diagnosis

\begin{abstract}
Background: With the development of new technologies such as magnifying endoscopy with narrow band imaging, endoscopists achieved better accuracy for diagnosis of gastric cancer (GC) in various aspects. However, to master such skill takes substantial effort and could be difficult for inexperienced doctors. Therefore, a novel diagnostic method based on artificial intelligence (AI) was developed and its effectiveness was confirmed in many studies. Al system using convolutional neural network has showed marvelous results in the ongoing trials of computer-aided detection of colorectal polyps. Summary: With Al's efficient computational power and learning capacities, endoscopists could improve their diagnostic accuracy and avoid the overlooking or over-diagnosis of gastric neoplasm. Several systems have been reported to achieved decent accuracy. Thus, Al-assisted endoscopy showed great potential on more accurate and sensitive ways for early detection, differentiation, and invasion depth prediction of gastric lesions. However, the feasibility, effec-
\end{abstract}

tiveness, and safety in daily practice remain to be tested. Key messages: This review summarizes the current status of different Al applications in early GC diagnosis. More randomized controlled trails will be needed before Al could be widely put into clinical practice.

(c) 2021 S. Karger AG, Basel

\section{Introduction}

Gastric cancer (GC) is the fifth most common cancer worldwide [1] and the third leading cause of cancer death. The incidence rate differs in various regions but the highest is among East Asian populations. Successful detection of early GC (EGC) could significantly improve survival up to $90 \%$ [2].

Endoscopy is the most powerful tool for detection and diagnosis of GC, but the accuracy of detection depends on the experience of the endoscopists and is complicated by various factors of the gastrointestinal (GI) tract. Accordingly, advanced endoscopic techniques such as image-enhanced endoscopy have been developed to improve the detection of EGC. Still, the false negative rate of GC detected by esophagogastroduodenoscopy (EGD) 
was reported to be between $4.6 \%$ and $25.8 \%$ [3-5]. More accurate and objective methods for EGC detection are desired for clinical practice. As for advanced technics pertaining diagnosis of EGC, such as prediction of invasion depth and differentiation from non-neoplasm, the accuracy differs drastically between experts and trainees.

Recently, artificial intelligence (AI) has caught considerable attention in various medical fields. In the field of gastroenterology, AI applications in capsule endoscopy [6] and in the detection, localization, and segmentation of colonic polyps [7] have been reported as well. Like described by Hsiao et al. [8], AI could be applicated in assisting the decision of biopsy and ESD evaluation. Among the different AI models, convolutional neural network $(\mathrm{CNN})$ is a method most commonly used in medical imaging [9].

As an AI algorithm that automatically learns features from the data, CNN has been utilized mainly for image recognition [10]. It is designed to think similarly to the human brain using huge datasets of image to learn certain patterns. That is to say, $\mathrm{CNN}$ is ideal for endoscopic image recognition to detect and localize GI neoplasms. Characteristic of neoplasm were learned using endoscopic images previously confirmed by endoscopists. After training, the CNN will be tested on non-labeled external datasets and determine whether the model could correctly identify previously unseen neoplasms. Hopefully, the algorithm can identify what it believes is a gastric neoplasm in a real-time endoscopic video input and predict the invasion depth. We try to review recent developments about the application of $\mathrm{AI}$ in the diagnosis of EGC.

\section{Detection}

The detection of EGC and precancerous neoplasms is essential for the minimum invasive resection and, thus improves prognosis. Since EGC can only have very subtle differences when compared with surrounding mucosa, detection for inexperienced endoscopists could be challenging. Thus, it is paramount to improve the detection rates of EGC using new methods. Many groups have reported to already started integrating $\mathrm{AI}$ into their routine practice to improve the overall detection rates of GC.

Sakai et al. [11] proposed a CNN-based automatic detection scheme to assist the diagnosis of EGC in endoscopic images. Transfer learning was performed using 2 classes (cancer and normal) of image datasets that have detailed texture information on lesions derived from a small number of annotated images. The accuracy of trained network was $87.6 \%$, while the detection accuracy of external dataset was $82.8 \%$.

Hirasawa et al. [12] constructed an AI-based diagnostic system that was trained by $>13,000$ images of EGD to detect early and advanced GC (AGC), and then tested the diagnostic accuracy. The $\mathrm{CNN}$ had an overall sensitivity of $92.2 \%$, and a positive predictive value (PPV) of $30.6 \%$. Seventy of the 71 lesions (98.6\%) with a diameter of $6 \mathrm{~mm}$ or more as well as all invasive cancers were correctly detected. The diagnostic ability of the same system was compared by Ikenoyama et al. [13] to that of 67 endoscopists using an independent test dataset (2,940 images from 140 cases). The average diagnostic time for analyzing 2,940 test endoscopic images by the $\mathrm{CNN}$ and endoscopists were $45.5 \pm 1.8 \mathrm{~s}$ and $173.0 \pm 66.0 \mathrm{~min}$, respectively. The $\mathrm{CNN}$ had a significantly higher sensitivity than the endoscopists (by 26.5\%; 95\% CI, 14.9-32.5\%). This study was limited to early GC lesions smaller than 20 $\mathrm{mm}$ and difficult to detect.

One real-time assistance system developed by $\mathrm{Wu}$ et al. [14] ensure that the whole stomach is observed during endoscopy without blind spot, thereby providing a prerequisite for the detection of EGC. This system identified EGC from non-malignancy with an accuracy of $92.5 \%$, a sensitivity of $94.0 \%$, a specificity of $91.0 \%$, a PPV of $91.3 \%$, and a negative predictive value (NPV) of $93.8 \%$, outperforming all levels of endoscopists. Later, the same group named this system as WISENSE (a combination of "wise" and 'sense') and conducted a randomized controlled trial to test its effectiveness for monitoring blind spots during EGD. WISENSE monitored blind spots with an accuracy of $90.40 \%$ in real EGD videos. A total of 324 patients were recruited and randomized, 153 and 150 patients were analyzed in the WISENSE and control group, respectively. Blind spot rate was lower in WISENSE group compared with the control $(5.86 \%$ vs. $22.46 \%, p<0.001)$ [15].

Gastrointestinal Artificial Intelligence Diagnostic System (GRAIDS) is another real-time AI system developed by Luo et al. [16]. 1,036,496 white light images from 84,424 individuals were used to develop and test GRAIDS. The diagnostic accuracy in identifying upper gastrointestinal cancers was $95.5 \%$ in the internal validation set and ranged from $91.5 \%$ to $97.7 \%$ in the 5 external validation sets. GRAIDS achieved diagnostic sensitivity similar to that of the expert endoscopists $(94.2 \%$ vs. $94.5 \%$; $p=$ $0.692)$ and superior sensitivity compared with competent $(85.8 \%, p<0.0001)$ and trainee $(72 \%, p<0.0001)$ endoscopists. 


\section{Prediction of Invasion Depth}

One of the most important preoperative criteria for curative endoscopic resection is the tumor invasion depth. Curative resection by ESD can frequently be achieved for intramucosal cancer (M) and cancer with submucosal invasion $<500 \mu \mathrm{m}$ (SM1), whereas deep invasive GC was required for radical surgery. Although the efficacy of using macroscopic features [17] and EUS [18] for prediction for invasion depth has been reported, a more accurate method is still warranted.

In this very first study for prediction of invasion depth using AI by Zhu et al. [19], an AI-based CNN system was developed through transfer learning leveraging a state-ofthe-art pretrained CNN architecture including $790 \mathrm{im}$ ages as development dataset and another 203 images as test dataset. The $\mathrm{CNN}$ system achieved significantly higher accuracy (by 17.25\%; 95\% CI, 11.63-22.59) and specificity (by $32.21 \%$; 95\% CI, 26.78-37.44) than human endoscopists.

Cho et al. [20] established one algorithm to predict submucosal invasion events of gastric neoplasm based on endoscopic images. In the external validation, the mean areas under the curves (AUC) reached 0.887 (0.8630.910 ) which suggested potential clinical relevance during the choice of therapeutic strategy.

For differentiation between M-SM1 and SM2-SI invasive cancer, Nagao et al. [21] used 16,557 images from 1,084 cases of GC to develop an AI system through transfer learning leveraging a $\mathrm{CNN}$ architecture, ResNet5. The lesion-based sensitivity, specificity, accuracy, PPV, and NPV of this AI system were 84.4\%, 99.4\%, 94.5\%, 98.5\%, and $92.9 \%$, respectively.

Another study from Korea showed AUC of receiver operating characteristic curves for EGC detection and depth prediction were 0.981 and 0.851 , respectively. Among the factors affecting AI prediction of tumor depth, only histologic differentiation was significantly associated, where undifferentiated-type histology exhibited a lower AI accuracy [22].

\section{Differentiation of Neoplasm and Non-Neoplasm}

An accurate differentiation of gastric neoplasm and non-neoplasm is sometimes difficult with conventional white light imaging endoscopy; nevertheless, it remains the standard endoscopic examination modality. Over the years, several methods have been used for the differentiation of gastric neoplasm including NBI with or without magnification. Efforts were also made to test the efficacy of these methods using AI system. Some results indicate that by using magnifying endoscopy with narrow band imaging (ME-NBI), AI system could achieve satisfying diagnostic efficacy.

Horiuchi et al. [23] developed a 22-layer CNN system which was pretrained using 1492 EGC and 1,078 gastritis images from ME-NBI. This system achieved high sensitivity and speed for differentiation of EGC and gastritis. The accuracy of the CNN system with ME-NBI images was $85.3 \%$, with 220 of the 258 images being correctly diagnosed. The method's sensitivity, specificity, PPV, and NPV were $95.4 \%, 71.0 \%, 82.3 \%$, and $91.7 \%$, respectively. The overall test speed was 51.83 images/s (0.02 s/image).

Ueyama et al. [24] constructed an AI-assisted CNN computer-aided diagnosis (CAD) system, based on 5,574 still ME-NBI images. The overall accuracy, sensitivity and specificity of this system were $98.7 \%, 98 \%$, and $100 \%$, respectively. All misdiagnosed images of EGCs were of lowquality or of superficially depressed and intestinal-type intramucosal cancers that were difficult to distinguish from gastritis, even by experienced endoscopists.

Comparison between AI systems and experts has been done in several institutes. Zhang et al. [25] constructed a CNN-based diagnostic system based on a ResNet34 residual network structure and a DeepLabv3 structure using 21,217 non-magnified white light images of 5 gastric conditions, peptic ulcer, EGC, and high-grade intraepithelial neoplasia (HGIN), AGC, gastric submucosal tumors, and normal gastric mucosa without lesions. After comparing with experienced endoscopists, the diagnostic specificity and PPV of the CNN were higher than that of the endoscopists for the EGC and HGIN images (specificity: $91.2 \%$ vs. $86.7 \%$, by $4.5 \%, 95 \%$ CI, $2.8-7.2 \%$; PPV: $55.4 \%$ vs. $41.7 \%$, by $13.7 \%, 95 \%$ CI, $11.2-16.8 \%)$ and the diagnostic accuracy of the $\mathrm{CNN}$ was close to those of the endoscopists for the lesion-free, EGC and HGIN, peptic ulcer, AGC, and submucosal tumors images.

$\mathrm{Li}$ et al. [26] retrospectively reviewed 386 images of noncancerous lesions and 1,702 images of EGC for CNN training and then prospectively enrolled 171 images of noncancerous lesions and 171 images of EGC to test and evaluate the diagnostic capability of CNN. The sensitivity, specificity, and accuracy of CNN system in the diagnosis of EGC were $91.18 \%, 90.64 \%$, and $90.91 \%$, respectively. No significant difference was spotted in the specificity and accuracy of diagnosis between $\mathrm{CNN}$ and experts but those were significantly higher than those of the nonexperts.

Besides still images of ME-NBI, using video clips during magnification as pre-training material may also contribute to improve the efficacy of AI system. One CAD system was pretrained using 2,570 still images (1,492 can- 


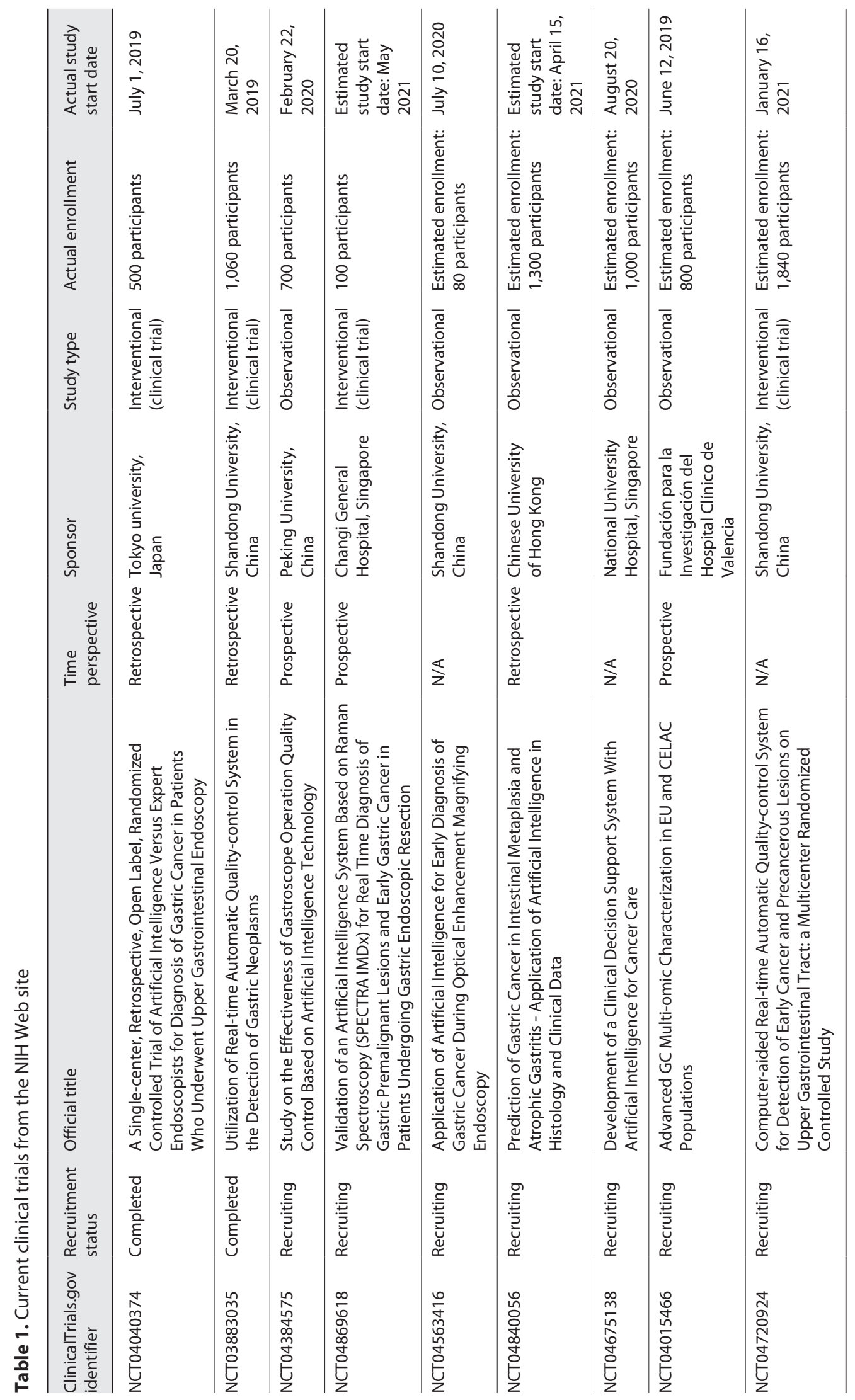


cerous and 1,078 noncancerous images) and 174 videos (87 cancerous and 87 noncancerous videos) obtained using ME-NBI. Horiuchi et al. [27] used this system to compare with 11 experts who were skilled in diagnosing EGC using ME-NBI with clinical experience. The CAD system demonstrated an AUC of 0.8684 . The accuracy, sensitivity, specificity, PPV, and NPV were $85.1 \%$ (95\% CI, 79.089.6), 87.4\% (95\% CI, 78.8-92.8), 82.8\% (95\% CI, 73.589.3), 83.5\% (95\% CI, 74.6-89.7), and $86.7 \%$ (95\% CI, 77.8-92.4), respectively, which were equivalent to or better than that of several experts.

\section{Pathological Diagnosis}

The histological examination for large ESD specimen could be time-consuming and requires additional effort for the evaluation of risk factor like lymphavascular invasion. Song et al. [28] reported a clinically applicable system using a deep CNN trained with 2,123 pixel-level annotated H\&E-stained whole slide images. This model achieves a sensitivity near $100 \%$ and an average specificity of $80.6 \%$ on a real-world test dataset with 3,212 whole slide images digitalized by 3 scanners. This system showed some promise for aiding pathologists in improvement of diagnostic accuracy and prevention of misdiagnoses.

\section{Ongoing Clinical Trials and Possible Future Path}

Although a bunch of researches on developing CNN systems has been published recently [29], the relatively lacking of RCT has always been a holdback. When searching PubMed for AI and endoscopic procedures, only a limited number of RCT enlisted pertaining EGC could be found, casting a doubt on the future of integrating AI into clinical practice.

To further elaborate this topic, we then performed search on https://clinicaltrials.gov/ with the term "Artificial Intelligence" and "Gastric Cancer" [30]. Only 9 clinical trials are currently listed, and 7 of them are still recruiting (Table 1). Nation-wise, most of these trials are on far-east Asian, either Japan or China. This is quite reasonable due to high prevalence of GC in these regions.

Among the registered trials, only 3 trials are labeled as prospective. Amongst them 2 trials were still recruiting, while one research in China led by Yanqing Li has prospective RCT in esophagus and colon polyps, instead of EGC. It is possible they have unpublished materials that may emerge in the near future.

In China, Tang et al. [31] reported a retrospective research of a DCNN system for detecting EGC, with an external validation dataset from other institutions. Training of the system was conducted by 35,823 images from 1,085 patients, the system was then validated by 9,417 images from 279 patients. Validation dataset obtained from other institutions was then applied on the system as external validation, showing a promising result of high overall performance, with a PPV of $86.9 \%$, an NPV of $90.7 \%$, and an AUC of 0.906 . The system was then applied on OGD videos, showing a real time-based diagnostic ability with decent sensitivity. The authors stated a plan for future prospective RCT and also offered open access to their system.

In Japan, as mentioned earlier, Hirasawa et al. [12] also reported a retrospective RCT, in which the CNN system showed ability to process endoscopic images with a decent diagnostic ability. As an attempt to compare the diagnostic ability of the system, the same group of authors conducted a test to compare it with expert endoscopists [32].

Horiuchi et al. [13] conducted a retrospective research in which they constructed a CAD system that could be compared with expertise of seasoned endoscopists. The system was further tested under a single-center retrospective trial to further elaborate its possible usage under actual clinical usage [27]. It is also mentioned that they were planning a multicenter and prospective study differentiating gastritis and GC through a biopsy of all cases.

However, all of the research works mentioned above are retrospective, which are prone to bias. This means future RCT is required before AI could be put into actual clinical usage.

Another possible holdback hides in the underlining different algorithm. Although most research adapted $\mathrm{CNN}$ as their system of choice, different institutions have chosen to develop their own algorithm. This might be beneficial as diversity could bring game-changers to this world, but we must keep in mind that training of these AI system requires relatively large number of inputs, and we might be doing redundant labor during the process.

\section{Conclusion}

As described in this review article, many studies have already been published as stepping-stones toward the application of AI in diagnosing EGC. Some systems even showed high accuracy which could be compared with those of experts. However, before AI systems were verified by multicenter RCTs, it seems reasonable to only use AI for auxiliary diagnosis, like determining whether there is a blind spot during the process of EGD. After screening using high sensitivity system, suspicious lesions need to 
be further confirmed by experienced endoscopists. Falsenegative results will be the worst-case scenario in clinical practice because it means either EGC is not successfully detected, or additional surgery is needed. "Eyes can only see what brain knows." After enough information being inputted into the AI system and validation by multicenter RCTs, we should believe AI will have a better performance for EGC diagnosis.

\section{Conflict of Interest Statement}

The authors declare no conflicts of interest regarding this review article.

\section{Funding Sources}

This review was supported by a grant from the scientific research programs of Shanghai Municipal Commission of science and technology (No. 19411951504).

\section{Author Contributions}

Zili Xiao, Danian, Ji, and Feng Li did literature search and wrote the manuscript. Zhengliang Li and Zhijun Bao revised the manuscript.

\section{References}

1 Siegel RL, Miller KD, Jemal A. Cancer statistics, 2019. CA Cancer J Clin. 2019;69(1):7-34

2 Amin MB, Greene FL, Edge SB, Compton CC, Gershenwald JE, Brookland RK, et al. The eighth edition AJCC cancer staging manual: continuing to build a bridge from a population-based to a more "personalized" approach to cancer staging. CA Cancer J Clin. 2017; 67(2):93-9.

3 Menon S, Trudgill N. How commonly is upper gastrointestinal cancer missed at endoscopy? A meta-analysis. Endosc Int Open. 2014;2(2):E46-50.

4 Hosokawa O, Hattori M, Douden K, Hayashi H, Ohta K, Kaizaki Y. Difference in accuracy between gastroscopy and colonoscopy for detection of cancer. Hepatogastroenterology. 2007;54(74):442-4.

5 Voutilainen ME, Juhola MT. Evaluation of the diagnostic accuracy of gastroscopy to detect gastric tumours: clinicopathological features and prognosis of patients with gastric cancer missed on endoscopy. Eur J Gastroenterol Hepatol. 2005;17(12):1345-9.

6 Xia J, Xia T, Pan J, Gao F, Wang S, Qian YY, et al. Use of artificial intelligence for detection of gastric lesions by magnetically controlled capsule endoscopy. Gastrointest Endosc. 2021;93(1):133-e4.

7 Wang KW, Dong M. Potential applications of artificial intelligence in colorectal polyps and cancer: recent advances and prospects. World J Gastroenterol. 2020;26(34):5090-100.

8 Hsiao YJ, Wen YC, Lai WY, Lin YY, Yang YP, Chien Y, et al. Application of artificial intelligence-driven endoscopic screening and diagnosis of gastric cancer. World J Gastroenterol. 2021 Jun 14;27(22):2979-93.

9 Litjens G, Kooi T, Bejnordi BE, Setio AAA, Ciompi F, Ghafoorian M, et al. A survey on deep learning in medical image analysis. Med Image Anal. 2017;42:60-88.
10 Ebigbo A, Palm C, Probst A, Mendel R, Manzeneder J, Prinz F, et al. A technical review of artificial intelligence as applied to gastrointestinal endoscopy: clarifying the terminology. Endosc Int Open. 2019;7(12):E1616-E23.

11 Sakai Y, Takemoto S, Hori K, Nishimura M, Ikematsu H, Yano T, et al. Automatic detection of early gastric cancer in endoscopic images using a transferring convolutional neural network. Annu Int Conf IEEE Eng Med Biol Soc. 2018;2018:4138-41.

12 Hirasawa T, Aoyama K, Tanimoto T, Ishihara S, Shichijo S, Ozawa T, et al. Application of artificial intelligence using a convolutional neural network for detecting gastric cancer in endoscopic images. Gastric Cancer. 2018; 21(4):653-60.

13 Ikenoyama $Y$, Hirasawa T, Ishioka M, Namikawa K, Yoshimizu S, Horiuchi Y, et al. Detecting early gastric cancer: comparison between the diagnostic ability of convolutional neural networks and endoscopists. Dig Endosc. 2021;33(1):141-50.

14 Wu L, Zhou W, Wan X, Zhang J, Shen L, Hu $S$, et al. A deep neural network improves endoscopic detection of early gastric cancer without blind spots. Endoscopy. 2019;51(6): 522-31.

15 Wu L, Zhang J, Zhou W, An P, Shen L, Liu J, et al. Randomised controlled trial of WISENSE, a real-time quality improving system for monitoring blind spots during esophagogastroduodenoscopy. Gut. 2019;68(12): 2161-9.

16 Luo H, Xu G, Li C, He L, Luo L, Wang Z, et al. Real-time artificial intelligence for detection of upper gastrointestinal cancer by endoscopy: a multicentre, case-control, diagnostic study. Lancet Oncol. 2019;20(12):1645-54.

17 Abe S, Oda I, Shimazu T, Kinjo T, Tada K, Sakamoto T, et al. Depth-predicting score for differentiated early gastric cancer. Gastric Cancer. 2011;14(1):35-40.
18 Pei Q, Wang L, Pan J, Ling T, Lv Y, Zou X. Endoscopic ultrasonography for staging depth of invasion in early gastric cancer: a meta-analysis. J Gastroenterol Hepatol. 2015; 30(11):1566-73.

19 Zhu Y, Wang QC, Xu MD, Zhang Z, Cheng J, Zhong YS, et al. Application of convolutional neural network in the diagnosis of the invasion depth of gastric cancer based on conventional endoscopy. Gastrointest Endosc. 2019; 89(4):806-e1.

20 Cho BJ, Bang CS, Lee JJ, Seo CW, Kim JH. Prediction of submucosal invasion for gastric neoplasms in endoscopic images using deeplearning. J Clin Med. 2020 Jun 15;9(6):1858.

21 Nagao S, Tsuji Y, Sakaguchi Y, Takahashi Y, Minatsuki C, Niimi K, et al. Highly accurate artificial intelligence systems to predict the invasion depth of gastric cancer: efficacy of conventional white-light imaging, nonmagnifying narrow-band imaging, and indigo-carmine dye contrast imaging. Gastrointest Endosc. 2020;92(4):866-e1.

22 Yoon HJ, Kim S, Kim JH, Keum JS, Oh SI, Jo $\mathrm{J}$, et al. A lesion-based convolutional neural network improves endoscopic detection and depth prediction of early gastric cancer. J Clin Med. 2019 Aug 26;8(9):1310.

23 Horiuchi Y, Aoyama K, Tokai Y, Hirasawa T, Yoshimizu S, Ishiyama A, et al. Convolutional neural network for differentiating gastric cancer from gastritis using magnified endoscopy with narrow band imaging. Dig Dis Sci. 2020;65(5):1355-63.

24 Ueyama H, Kato Y, Akazawa Y, Yatagai N, Komori $\mathrm{H}$, Takeda T, et al. Application of artificial intelligence using a convolutional neural network for diagnosis of early gastric cancer based on magnifying endoscopy with narrow-band imaging. J Gastroenterol Hepatol. 2021;36(2):482-9. 
25 Zhang L, Zhang Y, Wang L, Wang J, Liu Y. Diagnosis of gastric lesions through a deep convolutional neural network. Dig Endosc. 2021;33(5):788-96.

26 Li L, Chen Y, Shen Z, Zhang X, Sang J, Ding $Y$, et al. Convolutional neural network for the diagnosis of early gastric cancer based on magnifying narrow band imaging. Gastric Cancer. 2020;23(1):126-32.

27 Horiuchi Y, Hirasawa T, Ishizuka N, Tokai Y, Namikawa K, Yoshimizu S, et al. Performance of a computer-aided diagnosis system in diagnosing early gastric cancer using magnifying endoscopy videos with narrow-band imaging (with videos). Gastrointest Endosc. 2020; 92(4):856-e1.
28 Song Z, Zou S, Zhou W, Huang Y, Shao L, Yuan J, et al. Clinically applicable histopathological diagnosis system for gastric cancer detection using deep learning. Nat Commun. 2020;11(1):4294.

29 Jin P, Ji X, Kang W, Li Y, Liu H, Ma F, et al. Artificial intelligence in gastric cancer: a systematic review. J Cancer Res Clin Oncol. 2020 Sep;146(9):2339-50.

30 National Library of Medicine (NLM) at the National Institutes of Health (NIH) [Internet]. Search result [cited 2021 Feb 10]. Available from: https://clinicaltrials.gov/ ct $2 /$ results?term $=$ Artificial+Intelligence $\&$ cond $=$ Gastric + Cancer $\&$ draw $=2 \&$ rank $=$ 9\#rowId8.
31 Tang D, Wang L, Ling T, Lv Y, Ni M, Zhan Q, et al. Development and validation of a realtime artificial intelligence-assisted system for detecting early gastric cancer: a multicentre retrospective diagnostic study. EBioMedicine. 2020 Dec;62:103146.

32 Hirasawa T, Ikenoyama Y, Ishioka M, Namikawa K, Horiuchi Y, Nakashima H, et al. Current status and future perspective of artificial intelligence applications in endoscopic diagnosis and management of gastric cancer. Dig Endosc. 2021 Jan;33(2):263-72. 